Irmtraud Behr

\title{
Semantische Aspekte des \\ Sauberkeitsdiskurses im öffentlichen Raum, am Beispiel von Mülleimeraufschriften
}

\section{Einleitung $^{1}$}

\subsection{Die Situation}

(A) Ein Passant wirft einen Gegenstand in einen Müllkorb.

Dieser Satz beschreibt eine alltägliche Handlung in einem urbanen Umfeld. Die Handlung selbst wird sehr häufig ausgeführt - aber leider oft auch nicht ausgeführt. ${ }^{2}$ Statt im Müllkorb landet Abfall auf Straßen und Plätzen oder in Grünanlagen. Eine solche Situation ist aus verschiedenen Gründen nicht erwünscht, und Stadtverwaltungen ergreifen eine Reihe von Maßnahmen, um die Bürger zu einer verantwortlichen Haltung anzuregen. Konzertierte Kampagnen zielen darauf ab, die Bürger zu sensibilisieren. Die Kampagnen verbinden Plakate, Werbung auf Müllautos und die Betextung von Mülleimern. Die meisten Kampagnen setzen auf Humor und Aufklärung. ${ }^{3}$ Auf Plakaten und Müllautos werden die Bürger direkt angesprochen (1) oder die Bemühungen der Angestellten der jeweiligen Stadtverwaltungen gepriesen (2):

(1) Du hast es in der Hand. (www.baukeinenmist.at, Stand: 23.11.2017)

(2) We kehr for you (BSR, Berlin) [Das Bild zeigt zwei Angestellte in Arbeitskleidung mit Besen in der Hand]

1 Ich danke Colette Cortès, Heike Romoth, Vilmos Ágel sowie Pierre-Yves Modicom für ihre konstruktive und anregende Kritik an früheren Versionen dieses Aufsatzes.

2 Grill- und Picknicksaison: Ärger um Müll im grünen Idyll, Badische Zeitung, 26.5.2017. Siehe auch Breitbarth/Urban (2014).

3 Siehe die Geschäftsberichte verschiedener Stadtreinigungsämter und die Berichterstattung über Sauberkeitskampagnen (exemplarisch SPIEGELONLINE.de vom 22.6.2012, Radio Hamburg 27.3.2017). 
Ein anderer, komplementärer Weg besteht darin, die Mülleimer selbst $\mathrm{zu}$ betexten. Auf auffällig gestalteten Mülleimern sind Sprüche angebracht wie

(3a) Hamburgs kleinste Müllkippe. (Hamburg);

(3b) Mist für dich, Lob für mich. (Wien);

(3c) Man muss auch loslassen können (Innsbruck);

(3d) Ihre Papiere bitte! (in mehreren Städten).

Die Formulierungen beruhen auf Wortspielen. Das Ziel ist, dass die Passanten auf die urbanen Objekte „Mülleimer“ aufmerksam werden (Eggs 2013), dass also der pragmatische Blick auf die Umgebung, der lediglich einige relevante Elemente wahrnimmt, die zur Realisierung eines übergeordneten Zwecks notwendig sind, durch einen semantischen Blick abgelöst wird, der die materiellen, ästhetischen Aspekte privilegiert (Levitte 2013). Ein witziger Mülleimerspruch ist dafür ein geeignetes Mittel, da er nicht unmittelbar entschlüsselt wird. Der Passant muss zum Leser im eigentlichen Sinne werden, um einerseits den Sinn zu erfassen und andererseits den sprachspielerischen Mechanismus zu entdecken.

\subsection{Die Problematik}

Die vorliegende Untersuchung nimmt ihren Ausgang in der Beobachtung von sprachlich auffälligen Phänomen im öffentlichen Raum, die ich über Jahre hinweg in verschiedenen Städten und Ländern fotografiert habe. An die 50 Fotos von Mülleimersprüchen fügen sich so zu einer Belegsammlung zusammen, die Mülleimer als funktionale Objekte im urbanen Umfeld und als Textträger dokumentiert. Die Funktionalität kann handlungstheoretisch bestimmt werden: die Müllentsorgungshandlung als Element des urbanen, zivilisierten Verhaltens im öffentlichen Raum. Kleintexte im öffentlichen Raum zeichnen sich durch ihre Kürze und materielle Begrenztheit aus (Behr 2005; Behr/Lefeuvre eingereicht), und Mülleimer mutieren in dieser Hinsicht zu fast idealen Textträgern, erlauben sie doch die Anbringung von großformatigen, sloganhaftigen einfachen oder komplexen Ausdrücken. Bleibt, den spezifischen Beitrag der sprachlichen Gestaltung der Mülleimeraufschriften zu untersuchen.

Ein augenfälliger Untersuchungsaspekt sind die Wort- und Sprachspiele, die phraseologischen De- und Remotivierungen (Eggs 2013). Darüber hinaus ist ein anderer Aspekt zentral für das Verständnis der Mülleimeraufschriften, nämlich die Art und Weise, wie der witzige Mülleimerspruch auf die Müllentsorgungshandlung referiert: Was wird explizit versprachlicht, was wird mitbedeutet? Was steht im Vordergrund, was im Hintergrund? Auf diese Frage möchte ich mich hier 
konzentrieren. Dabei werde ich neutrale Mülleimeraufschriften mit in die Untersuchung einbeziehen, um die jeweiligen Spezifitäten herauszuarbeiten.

Ich betrachte die Kleinmüllentsorgungssituation als eine außersprachliche, sozial eingeübte Situation, die kognitiv mittels verschiedener Ereignisschemata (Pörings/Schmitz 2003) erfasst werden kann. Die außersprachliche Situation ist an sich unperspektiviert, erst das perzeptive, kognitive, vor allem aber das sprachliche Erfassen ${ }^{4}$ erfordert eine gewisse, schemageleitete und formulierungsabhängige Perspektivierung, ${ }^{5}$ also die Fassung von Referenzobjekten als Argumente bzw. Rollenträger in einer bestimmten Reihenfolge. ${ }^{6}$

Ich unterscheide die denotativ-semantische und die signifikativ-semantische Ebene (Welke 2011; Ágel 2017). Auf der denotativen Ebene kann eine gewisse Konstanz der Rollen angenommen werden (Ágel 2017: 6; mit Bezug auf Fillmore 1968) sowie eine gewisse Vollständigkeit der Rollenverteilung (Welke 2011: 144-148): Also der kleinmülldeponierende Passant als AGENs, der Kleinmüll als PATIENS, der Mülleimer wechselweise als ZIEL in einem Bewegungsschema oder als EMPFÄNGER in einem Übertragungsschema (Pörings/Schmitz 2003: 92-95). Auf der Ebene der einzelnen Ausformulierungen (also auf der signifikativ-semantischen Ebene) ist zu beachten, dass nicht zwingend alle drei Entitäten genannt werden müssen und dass die sprachlich indizierten Rollen variieren können. Die Kombination von Situationswissen und expliziten Elementen (Argumente, Prädikate) erlaubt es, über die signifikativ-semantischen neutralen oder witzigen Formulie-

4 Siehe dazu Köller (1993: 25), der die Rolle des Subjekts mit einbezieht: „Es ist offensichtlich, dass sich bei jeder Bildung eines konkreten Satzes oder Textes das Problem stellt, von welchem Sehepunkt aus ein Sachverhalt sprachlich objektiviert werden soll.“ Köller differenziert Aspekt, Sehepunkt und Perspektive. 'Aspekt' thematisiert die Grunderfahrung, „dass wir beim Sehen einen Gegenstand nie total erfassen, sondern nur hinsichtlich derjenigen Seite, die unserem jeweiligen Wahrnehmungsstandort zugewandt sind“; 'Sehepunkt' macht kenntlich, „dass jede konkrete Wahrnehmung von einer bestimmten räumlichen, zeitlichen und letztlich auch geistigen Position aus erfolgt“; der Sehepunkt bestimmt also die wahrnehmbaren Aspekte. 'Perspektive’ betont die „relationale Genese“ aller Wahrnehmungsinhalte, den Zusammenhang zwischen Objekts- und Subjektssphähre. „Perspektiven sind die Weisen, in denen die Subjekte in die Welt hineingleiten und Kontakt zu ihren Wahrnehmungsobjekten gewinnen.“

5 „Mithilfe von expliziten Prädikationen profilieren Sprachbenutzer und Sprachbenutzerinnen bestimmte Wissensaspekte eines Referenzobjektes, während andere in den Hintergrund treten. Jede Prädikation perspektiviert das Referenzobjekt auf eine bestimmte Art und Weise.“ (Ziem 2008: 331). ,Jede Repräsentation, ob perzeptiver oder sprachlicher Art, setzt Auswahl und eine bestimmte Perspektivierung voraus. Die Satztypen (Konstruktionstypen), die die Sprecher in ihrer Sprache vorfinden (und an deren ständiger Umformung sie mitwirken), stellen ihnen Perspektivierungen zur Verfügung.“ (Welke 2011: 84).

6 Siehe die Diskussion bei Welke (2011: 154-161). Auch Pörings/Schmitz (2003: 95) gehen von einer Perspektivierung der Teilnehmerrollen als erster, zweiter, dritter Teilnehmer aus. 
rungen, die semantischen Schemata zu erschließen und sie als direkte oder indirekte Handlungsanweisungen zu verstehen.

\section{Situationsbeschreibung und Verbalisierungen}

Die Mülleimersprüche sind Teil eines Sauberkeitsdiskurses (Auer 2010) in dem Sinne, dass sie mit den anderen sprachlichen und semiotischen Äußerungen ein ganzes Netz von Bedeutungen aufbauen. Die Besonderheit der Mülleimersprüche besteht nun darin, dass sie gezielt auf die Handlung referieren, die der vorbildliche Mitbürger ausführen soll, damit die Straßen, Plätze und Parks sauber, d.h. müllfrei bleiben. Ich möchte im Folgenden untersuchen, wie man dieses sprachliche Fokussieren auf die soziale Handlung modellieren kann.

\subsection{Der situationsbeschreibende Satz, Verwendungen und Varianten}

Gehen wir zurück zu dem eingangs zitierten Satz (A) Ein Passant wirft einen Gegenstand in einen Müllkorb. Eigentlich wird ein solcher Satz kaum geäußert. Er könnte vielleicht am Anfang eines Romans oder in der Beschreibung einer Straßenszene stehen, oder ein Beobachter an einem Fenster könnte ihn aussprechen, um andere Personen, die die Szene nicht beobachten können, zu informieren. Man begegnet Varianten des Satzes (A) in kritischen Texten, ${ }^{7}$ z.B. in Zeitungsartikeln, die Umweltsünden anprangern.

Auch wenn er kaum in der Form (A) geäußert wird, referiert der Satz doch auf eine alltägliche Situation. Das richtige Verhalten in Punkto Kleinmüllentsorgung wird im Laufe der Sozialisierung erlernt, u.a. auch durch Verbalisierungen wie „Jetzt werfen wir die Bananenschale in den Mülleimer da hinten“, mit denen Erwachsene den Kindern sowohl die Kategorisierung von Gegenständen als auch die dazugehörigen Handlungen beibringen. Die Erwachsenen versprachlichen

7 Unter der Überschrift „Die Anderen tun es ja auch. Warum Menschen ihren Abfall auf die Strasse werfen“ enthält der erste Abschnitt des Textes folgende Formulierung: „Unterwegs noch schnell einen Becher Kaffee hinunter gekippt oder einen Schokoladenriegel verputzt - doch wohin mit dem Abfall? Viele Passanten deponieren ihre lästig gewordenen Verpackungen an allen möglichen Stellen - nur nicht im Mülleimer.“ (Geiser 2010). 
die Handlung durch verschiedene situationsspezifische Äußerungen, denen referenzielle Konstanten zu Grunde liegen.

Die außersprachliche Situation kann auf verschiedene Weisen aufgerufen werden. Der Eingangssatz (A) referiert auf die mentale Repräsentation einer Situation, also auf Situationsbeteiligte mit ihren Eigenschaften und Relationen untereinander (Lehmann o.J.). ${ }^{8}$ Die Situation besteht aus drei Situationsbeteiligten (einem Handelnden (Passant) und zwei Objekten (Kleinmüll, Müllbehälter)) sowie einer zielgerichteten Handlung (Kleinmüll in Behälter deponieren). Die Realisierung dieser Situation wird in verschiedenen kommunikativen Situationen eingefordert, unter anderem durch die Mülleimeraufschriften. Die Aufschriften kennzeichnen sich sprachlich dadurch, dass sie a) nicht alle Situationsbeteiligten nennen, b) verschiedene Prädikationen realisieren. Sie geben also verschiedenen Perspektivierungen Ausdruck.

So enthält der Satz (A) ein Handlungsprädikat mit dem Passanten als grammatischem Subjekt. Daneben gibt es noch andere Sichtweisen auf die Situation, z.B. eine passivische, in der der mobile Gegenstand (der Kleinmüll) die Subjektrolle übernimmt:

(B) Ein Gegenstand wird [von einem Passanten] in den Mülleimer geworfen.

Der weggeworfene Gegenstand kann auch in aktivischen Formulierungen (4) und (5) als Subjekt fungieren: ${ }^{9}$

(4) „viele Dinge, die wir einmal benutzt haben, landen in der Tonne, ohne dass sie wiederverwendet werden“ (VON CHARLOTTE MEYER | 15.4.2016 14:09 Thermosflasche anstatt Pappbecher - Geht so eine Uni ohne Müll?)

(5) Mehr als 45 Millionen Tonnen Haushaltsmüll wandern in Deutschland jedes Jahr in die Tonne. (SZ.de, 11. Mai 2017) (www.sueddeutsche.de/muenchen/ muell-warum-muenchen-so-sauber-ist-1.3495828, Stand: 13.5.2017)

8 Lehmann (o.J.) definiert Situationen als eine „statische oder dynamische Konfiguration aus Situationsbeteiligten / Partizipanten“ „mit ihren Eigenschaften und Beziehungen zueinander“, die „im Prinzip durch einen Satz kodiert“ wird (Lehmann, o.J.). „Situationsbeteiligte werden als Partizipanten versprachlicht“; sie haben sowohl situationsunabhängige Eigenschaften, sie „tragen verschiedene semantische Rollen, die die Weise ihrer Beteiligung an der Situation bzw. ihre spezifische Beziehung zu den anderen Partizipanten verkörpern“.

9 „Viele Dinge / Hausmüll“ ist denotativ-semantisch PATIENS, aber signifikativ-semantisch VoRGANGSTRÄGER (in der Terminologie von Ágel 2017). 


\subsection{Eine spezifische Konstellation}

Mit Sätzen wie (4-5) wird aus einer aktivischen PATIEns-zentrierten Perspektive heraus mit verschiedenen Fokussierungen auf Situationen referiert. Es sind Sätze in fortlaufenden Texten. Eine andere Konstellation liegt vor, wenn Aufschriften auf Müllbehältern Anweisungen enthalten, wie der Müll zu entsorgen ist bzw. welche Art von Müll deponiert werden darf:

(6a) Nur Reiseabfälle (Aufschrift auf einer Mülltonne an einer Autobahnraststätte, Süddeutschland)

(6b) Braunglas (Aufschrift auf einer Glasabfallsammeltonne)

(7) Textil und paarweise gebündelte Schuhe bitte sauber und trocken, verpackt in Kunststofftüten, einwerfen (Aufschrift auf einer Textiltonne, Stadtreinigung Leipzig)

Auch diese Versprachlichungen sind Patiens-zentriert. Das im Eingangssatz (A) verbalisierte Verhalten muss als gewusst vorausgesetzt werden, wenn ein Leser (6-7) verstehen will. ${ }^{10}$ Die sprachlichen Instruktionen geben präzise Vorgaben für die korrekte Realisierung einer erwünschten Handlung, in diesem Falle die Kleinmüllentsorgung.

Diese Instruktionen sind relevant, wenn ein Agens mit einer bestimmten Intention (nämlich der, einen Gegenstand zu entsorgen) vor dem Müllbehälter steht und die Aufschrift liest. (6) ist die konzise, gut sichtbare Hauptbotschaft in einer entsprechenden Schriftgröße und enthält lediglich Bezeichnungen für die Gegenstände, die in den Behältern deponiert werden dürfen. (7) realisiert einen deontischen Infinitiv und enthält den Aufforderungsmarker bitte. Als Präzisierung der implizit eingeforderten Handlung ist (7) kleiner geschrieben und steht unterhalb von (6) oder auf einer anderen Seite des Müllbehälters. (6) ist also funktional den witzigen Mülleimeraufschriften äquivalent, indem es a) visuell äquivalent ist und b) wie die witzigen Sprüche über bestimmte Selektionen und Prädikationen auf Elemente der durch (A) verbalisierten Situation referiert. Im Folgenden wird also nur (6) bei den Vergleichen berücksichtigt.

10 In der Tat referiert (A) auf die drei Entitäten der Kleinmüllentsorgungssituation, im Gegensatz $\mathrm{zu}(4-7)$ und kann somit als die explizite Folie angesehen werden, auf der die verschiedenen Versprachlichungen interpretiert werden. 


\section{Perspektivierungen}

\subsection{Schematische Interpretation}

In den Sätzen (4-5) werden Situationen beschrieben, die sich anhand des allgemeinen Bewegungsschemas (Pörings/Schmitz 2003: 88) erfassen lassen. Das allgemeine Bewegungsschema ist eine Kombination aus einem Vorgangsschema bzw. einem Handlungsschema mit den Punkten Ursprung - Weg - Ziel, die alle gleich prominent sind und deshalb unabhängig voneinander zum Ausdruck gebracht werden können: Der Apfel fällt vom Baum (Vorgang, Ursprung), der Apfel fällt ins Gras (Vorgang, Ziel), Der Junge klettert auf den Baum (Handlung, Ziel hervorgehoben), der Junge klettert den Baum hinauf (Handlung, Weg hervorgehoben) (nach Pörings/Schmitz 2003: 88f.).

Der Eingangssatz (A) Ein Passant wirft einen Gegenstand in einen Müllkorb kann nun analysiert werden als Ein Passant macht, dass ein Gegenstand im Müllkorb landet, wobei das Ziel hervorgehoben ist. Er kann also als die agentivische Formulierung angesehen werden, das Verb werfen impliziert eine kontrollierende, bewegende Kraft, die den Gegenstand von seinem aktuellen Ort zum Ziel, d.h. ins Innere des Mülleimers bewegt. ${ }^{11}$ Das Bewegungsschema wird in ein kausatives Schema eingebettet: AgENS macht (PATIENs bewegt sich [von a] zum ZiEL).

\subsection{Selektionen}

Die Mülleimeraufschriften realisieren nun nicht die Gesamtheit dieser Bedeutungsstruktur, sondern instanziieren einzelne Positionen bzw. Elemente der semantischen Gesamtstruktur. Als „Gegenstand“ (PatiEns) kommen z.B. Reiseabfälle oder Braunglas (6) in Frage, was die neutralen Mülleimeraufschriften genau präzisieren.

Witzige Mülleimeraufschriften hingegen suggerieren eher die Arten von Gegenständen, die in ihnen entsorgt werden können: Trinkbecher (Becherbutler, Berlin) oder Papier (Ihre Papiere bitte!, mehrere Städte). Darüber hinaus fokussieren sie auch die Eigenschaften des Mülleimers an sich (Stille Eimer sind tief, Hamburg) in seiner Rolle als ZIEL. Sie können auch die Handlung selber fokussieren:

11 Siehe auch Wunderlich (2003: 1), der das dreistellige Verb werfen folgendermaßen repräsentiert: ACT(x) \& BECOM LOC(z,AT(y)), y ist ein Ziel. Dabei ist ACT(x) ein unspezifisches Prädikat und y das dritte Argument. 
Wirf es in den Eimer, Kleines (Hamburg) (Handlungsprädikat). Die Möglichkeiten sind also weiter gestreut als bei den neutralen Mülleimeraufschriften.

\subsection{Bewegungs-/Übertragungsschema}

Verschiedene signifikativ-semantische Formulierungen können auf das Bewegungsschema zurückgeführt werden:

$$
\text { Kippen gehören eingelocht! }{ }^{12} \text { (Hamburg) }
$$

Die Kippen gehören also ins Loch, d.h. jemand muss sie ins Loch bewegen.

(8) ist ein Beispiel für eine passivische Perspektivierung, mit Fokussierung auf dem PATIENS und modaler Einfärbung: Eine bestimmte Handlung wird eingefordert.

Aber die witzigen Mülleimeraufschriften zeigen noch ein anderes Schema:

$$
\text { Das Publikum gibt mir alles (Wien) }
$$

Eine solche Aufschrift realisiert nicht das Bewegungsschema, sondern das Übertragungsschema (Pörings/Schlitz 2003: 90). ${ }^{13}$ Beide Schemata sind dreiteilig, beide enthalten die denotativen Rollen Agens und PATIENS. Die dritte Rolle im Übertragungsschema ist jedoch nicht das ZIEL, oder besser: Es ist ein Ziel besonderer Art, nämlich ein EMPFÄNGER. Die Nähe der beiden Schemata erlaubt es,

12 Das gehören-Passiv ist eine Perspektivierung, die das Agens ausblendet. Hier ist das Ziel ins Verb inkorporiert. Einlochen hat zwei Bedeutungen (www.dwds.de/wb/einlochen, Stand: 13.5.2017): 1. (salopp) ins Gefängnis bringen, einsperren, 2. (Golf) (den Ball) in ein Loch spielen (dwds.de). Der Hamburger Müllbehälter ist mit einer runden Öffnung am oberen, waagerechten Teil versehen und weist somit Ähnlichkeit mit den ebenfalls horizontalen runden Löchern im Golfspiel auf. Das Verständnis dieses Spruchs erfordert einige Verschiebungen. Kann im konkreten Sinne eine Parallele zwischen dem Platzieren des Golfballs und dem Wegwerfen des Zigarettenstummels gezogen werden, so handelt es sich doch um zwei verschiedene Domänen. Bei der Erfassung des übertragenen Sinnes (der, nebenbei bemerkt, der erste ist, der im Wörterbuch angegeben wird) werden Konnotationen an Strafbarkeit erweckt, was durch die modale Komponente des gehören-Passivs unterstützt wird.

13 Nach Wunderlich (2003: 1) besteht eine der Varianten für semantisch dreistellige Prädikate darin, „das dritte Argument entweder als angezielten Possessor (Rezipient im engeren Sinne) oder als angezielte Lokation (Ziel im engeren Sinne) “ zu behandeln:
a. $\operatorname{ACT}(\mathrm{x})$ \& BECOME POSS $(\mathrm{y}, \mathrm{z})$
$\mathrm{y}$ ist ein Rezipent (geben)
b. ACT(x) \& BECOME LOC(z,AT(y))
$\mathrm{y}$ ist ein Ziel (werfen) 
eine Rezipientenperspektivierung ins Spiel zu bringen, die noch eine andere Erweiterung des Perspektivierungsspektrums möglich macht, nämlich eine Variation der Äußerungsinstanzen. ${ }^{14}$

\subsection{Variation der Äußerungsinstanzen}

Eine witzige Mülleimeraufschrift wie (9) realisiert das Übertragungsschema (Agens gibt EmpFänger PATIEns). Wer ist nun der EMPfänger? Der Rezeptionskontext bietet keinen natürlichen, d.h. menschlichen Kandidaten für diese Rolle. Der Mülleimer bietet sich an, falls man eine gewisse metaphorische Anthropologisierung akzeptiert. Die Aufschrift kann ihm auf Grund des Prinzips der lokalen Gültigkeit zugeschrieben werden, insofern als sie nur ,an und durch den Ort interpretierbar ist, an dem sie sich befindet“(Auer 2010: 276).

Die Platzierung auf der am besten sichtbaren Seite des Metallkörpers trägt dazu bei, einen lokalen Äußerungskontext zu konstituieren. So wie eine Aufschrift wie (6, Braunglas) für den Müllbehälter gültig ist, auf dem sie sich befindet, so konstruiert eine witzige Aufschrift wie (9, Das Publikum gibt mir alles) einen lokalen Äußerungskontext, in dem die durch mir instanziierte indexikalische Relation eine Zielreferenz finden kann.

Die eigentliche, unhintergehbare Äußerungsinstanz der Mülleimeraufschriften, seien sie witzig oder neutral, ist ein institutioneller Verfasser, der für die verschiedenen Inhalte und Formen verantwortlich zeichnet. ${ }^{15}$ Dies gilt auch, wenn witzige Mülleimeraufschriften per Aufruf gesammelt, von einer Jury bewertet und schließlich von der Stadtverwaltung angebracht werden.

Die Äußerungsinstanz kann nun zwischen verschiedenen Perspektivierungen wählen, zu denen auch die Übernahme bzw. Attribuierung der Sprecherrolle gehört. Als sprechendes Objekt kennzeichnet der Mülleimer sich selbst als

14 Eine solche mehrfache Perspektivierung spricht z.B. Welke (2011: 154) an: „Dass Menschen (und überhaupt Lebewesen) die Welt aus einer bestimmten Perspektive sehen, und zwar der ihren, ist etwas ganz und gar Elementares und Unhintergehbares. [...] Beim Menschen kommt die Fähigkeit der Einfühlung, der empathy hinzu [...], d.h. die Fähigkeit, etwas vom Standpunkt eines anderen aus zu sehen, auch eines Gegenstandes.“

15 Die verantwortlichen Institutionen sind stets vermerkt, Form und Platzierung des Vermerks können variieren. Die Berliner Stadtreinigung unterzeichnet ihre Kommunikation mit So orange ist nur Berlin BSR, gut sichtbar unter den Sprüchen oder an anderer Stelle auf den orangefarbenen Mülleimern. Die Hamburger Stadtreinigung platziert ihre Unterschrift Wir geben dem Müll einen Korb in der schwarzen Umrandung der Sprechblasen. Die Innsbrucker Stadtreinigung markiert schlicht Straßenbetrieb mitsamt einer Telefonnummer neben den Mülleimersprüchen. 
EMPFÄNGER und ZIEL einer Handlung (9). Die institutionelle Sprecherinstanz inszeniert ein sprechendes Objekt durch spezifische sprachliche Formen, nämlich die personale Deixis - die witzige Mülleimeraufschrift realisiert dann eine (quasi-)auktoriale Perspektive. Demgegenüber sind die nicht-deiktischen Aufschriften anderen Instanzen zuzuschreiben: einer allgemeinen Sprechergemeinschaft (8, Kippen gehören eingelocht!) oder einer neutralen, undifferenzierten Instanz $(10,11)$ :

(10) Kippendiener (Berlin)

(11) Beifall für Abfall (Wien)

Es sind also verschiedene Fokussierungen möglich sowie verschiedene signifikativ-semantische Perspektivierungen, die Diathese und Variationen der inszenierten Äußerungsinstanzen einschließen (Àgel 2017: 7f., 39-42). Den witzigen Mülleimeraufschriften liegen zwei semantische Handlungsschemata zugrunde, was das Spiel der inszenierten Äußerungsinstanzen befördert, indem der Mülleimer die denotative Rolle ZIEL oder EMPFÄNGER einnehmen kann.

\subsection{Prädikationen}

Die witzigen Mülleimeraufschriften realisieren mehrere Prädikationstypen. Die erste Klasse von Aufschriften enthält vor allem Bezeichnungen und Umbenennungen.

(12) Becherbutler (Berlin)

(13) Putzdamer Platz (Berlin )

(14) Hamburgs kleinste Müllkippe (Hamburg)

(15) Annahmestelle (Hamburg)

Zweierlei ist an diesen Aufschriften bemerkenswert. Sie realisieren eine Identifikations- (14) oder Kategorisierungsprädikation $(12,15)$ über das Objekt, auf dem sie sich befinden, d.h. den Mülleimer als ZIEL oder EMPFÄNGER. ${ }^{16}(12,14,15)$ könnten

16 (12, 14, 15) realisieren ein Essivschema (Pörings/Schmitz 2003: 83) über ein Element der Situation, die durch den Eingangssatz (A) beschrieben wird, erhöhen also punktuell die Informationsdichte im Gesamtschema. 
auch als Selbstbezeichnung interpretiert werden, also in einer (quasi-)auktorialen Perspektive. (13) hingegen referiert auf die unmittelbare urbane Umgebung des Mülleimers. Dabei weckt das Wortspiel Potsdamer/Putzdamer Platz Assoziationen an die Bedeutungen von putzen, wie ,reinigen, säubern, glänzend machen, (sich) schmücken, festlich kleiden, schönmachen“ (www.dwds.de/wb/putzen, Stand: 13.5.2017) und dockt damit an die situationsübergreifende Kleinmüllentsorgungsproblematik an.

Man findet auch explizite Eigenschaftsprädikationen, die sich auf den Mülleimer, seine Beschaffenheit und seine Charakteristiken beziehen:

(16) Habe durchgehend offen (Innsbruck)

(17) Für Sie rund um die Uhr geöffnet (Wien)

(18) Stille Eimer sind tief (Hamburg)

(19) Bin ned haglich ${ }^{17}$ (Wien)

(16-18) prädizieren Eigenschaften, die direkt sichtbar sind und verstoßen damit gegen die Grice'sche Quantitätsmaxime. Gerade darauf beruht der Überraschungseffekt, der zur reflexiven Bearbeitung einlädt. (19) behauptet eine Eigenschaft, die nicht direkt sichtbar ist, aber ebenfalls dem lesenden Passanten eine Interpretationsleistung abfordert: Welche Folgerungen kann man aus der Behauptung des Ned-haglich-Sein ziehen?

Eine andere Serie von witzigen Mülleimeraufschriften betont die EMPFÄNGERRolle:

(20) Haste mal ne Kippe? (Mannheim)

(21) Darf ich Ihnen etwas abnehmen? (Wien)

(22) Asche in mein Haupt (Hamburg)

(23) Bitte füttern (Berlin, Wien, Hamburg)

(20-22) enthalten deiktische Elemente (Personalpronomen der 1. und 2. Person, Possessivpronomen), die eine (quasi-)auktoriale Perspektivierung ausdrücken.

17 Haglich bedeutet heikel, wählerisch, schwer zu befriedigen (www.ostarrichi.org/dictionarysearch.html?search=haglich, Stand: 13.5.2017); heikel, (meist verwendet als) Eigenschaft einer Person, z.B. bei der Wahl von Speisen (www.fim.uni-linz.ac.at/Woerterbuch_oesterr_deut_ englisch.htm, Stand: 13.5.2017). 
Darüber hinaus instanziieren (20-23) das Übertragungsschema: Ein Gegenstand (Zigarettenkippe, Asche, unbestimmter Kleinmüll) wechselt aus der Sphäre des Passanten in die des Mülleimers.

Eine letzte Serie inszeniert Aspekte der Handlung des Übertragens bzw. des Ortswechsels selbst:

(24) Treffer, versenkt! (Hamburg)

(25) Wirf es in den Eimer, Kleines. (Hamburg)

(26) Man muss auch loslassen können (Innsbruck)

Diese Serie könnte um (8, Kippen gehören eingelocht!) und (23) erweitert werden. Die Verben fokussieren die Art und Weise $(8,23,25)$ bzw. einzelne Momente (24, 26) der komplexen Aktion, die der Passant ausführt, um einen Gegenstand wegzuwerfen.

Zusammenfassend kann man festhalten, dass die Prädikationen auf verschiedene Elemente des Bewegungs-/Übertragungsschemas zielen: den Mülleimer als EMPFÄNGER und ZIEL einer Wegwerf-Handlung, einzelne Aspekte eben dieser Handlung. Die prädikative Ebene komplettiert die denotativ perspektivische Ebene in der Gestaltung der witzigen Mülleimersprüche.

\section{Referenzausdrücke}

\subsection{Typologie und Bezeichnung der zu entsorgenden Gegenstände}

Die neutralen Mülleimeraufschriften verwenden sehr präzise Bezeichnungen für die zu entsorgenden Gegenstände: Textilien, Weiß-/Grün-/Braunglas, Restmüll, Pappe, Papier, Reiseabfälle, usw. Dadurch soll z.B. Mülltrennung gesichert werden, was die Weiterbearbeitung und Wiederaufbereitung des Mülls erleichtert. Die Bürger sind aufgefordert, die vergesellschafteten Anstrengungen für eine saubere Stadt und Umwelt zu unterstützen, d.h. einen Teil der Arbeit der Abfallwirtschaft zu übernehmen. Wenn sie Gegenstände in den spezialisierten Mülleimern oder Mülltonnen deponieren, handelt es sich um geplante, vorbereitete Handlungen.

Die witzigen Mülleimeraufschriften haben eine komplementäre Funktion: Die Mülleimer auf den Plätzen, Straßen oder in den Parks sind Entsorgungsstellen für den ungeplanten Abfall, bzw. für Abfallarten, die rasch entsorgt werden 
müssen. Es handelt sich also um Zigarettenabfälle (Zigarettenstummel, Asche), Hundekot, Kaugummi, Flaschen und Getränkedosen, anderen Kleinmüll wie Essensreste, Verpackungspapier, usw. Für einige Müllarten gibt es besondere Vorkehrungen: spezielle Container für Flaschen und Getränkedosen; Verteiler für Plastiktüten für Hundekot sowie entsprechende Container; Aschenbecher bzw. Zigarettenschächte (Hamburg) oder Tschickstummel (Wien). Der kleine Restmüll wird im Allgemeinen weder sprachlich differenziert noch abfallmäßig getrennt. Es begegnen Ausdrücke wie Abfall, Müll, Mist (Wien), Restmaterie, alles, Dreck, Papier (bzw. Papiere), seltener auch Becher, Zigarette, Asche, Kippe/Tschick. Die Besonderheit ist jedoch nicht so sehr die relative Unspezifik der Lexeme, sondern die Tatsache, dass sie in Ausdrücke größeren Umfangs und größerer Komplexität eingegliedert sind.

\subsection{Wortbildungsprodukte}

Lexeme, die auf eine der Müllarten verweisen, sind einfache oder komplexe Lexeme, die auch Bestandteile von Wortbildungsprodukten sein können:

(27) Beifall für Abfall (Wien)

(28) Kippendiener (Berlin)

(29) Schwarzes Loch sucht Restmaterie (Wien)

Diesen Beispielen kann man noch die Belege (12, Becherbutler) und (14, Müllkippe) hinzufügen. $(12,14,28)$ sowie $(29$, Restmaterie) sind zweigliedrige nominale Komposita, deren Erstglied auf verschiedene Arten von Abfall verweist. Müll als allgemeine Bezeichnung, Kippe als spezifische Bezeichnung sind eindeutig im Lexikon ausgewiesen. Gleiches gilt für Abfall als Ergebnis einer deverbalen Nominalisierung mit der Bedeutung ,(unverwertbarer) Überrest, der bei der Arbeit entsteht“ (www.dwds.de/wb/Abfall, Stand: 13.5.2017).

Becher hingegen ist nicht lexikalisch als Müll oder Abfall bestimmt (diese Bedeutung kommt im Lexikon nicht vor), sondern über das Wissen um soziale Praktiken. Erst dadurch, dass bestimmte Handlungen wie Essen und Trinken in den öffentlichen urbanen Bereich verlagert und in andere Handlungen eingegliedert sind, stellt sich die Frage nach der Entsorgung von Utensilien, die den mobilen Verzehr von Getränken und Speisen ermöglichen.

Rest bezeichnet wie Abfall „etwas, was beim Verbrauch oder Verzehr von etwas übrig geblieben ist“ (www.dwds.de/wb/Rest, Stand: 13.5.2017) und ist somit ein guter Kandidat für die Bezeichnung unspezifischen Kleinmülls. Rest- 
materie hingegen gehört zu verschiedenen Fachsprachen (Astronomie, Elektrostatik, Elementarphysik), und der Spruch (29) spielt natürlich mit den Bedeutungen und Referenzpotenzialen, die sich aus der Überlagerung der Diskursdomänen ergeben. In der Tat kann Restmaterie als transparente Komposition auch auf alltägliche materielle Objekte angewandt werden, während Schwarzes Loch neben der astronomischen Referenz auch die runde Öffnung des Müllkorbs (der innen dunkel ist) bezeichnet.

Als Erstglied eines nominalen Kompositums sind Becher, Kippe, Müll, Rest zwar Bezeichnungen für Abfall, sie sind jedoch keine unmittelbaren Konstituenten der witzigen Mülleimeraufschriften. Ein Kippendiener oder Becherbutler ist eine dienende Person, die für die Entsorgung von Kippen oder Bechern verantwortlich ist. Eine Restmaterie ist eine bestimmte Art von Materie, in diesem Zusammenhang eine, derer man sich entledigen will. Die Erstglieder lokalisieren die nominalen Basen in dem semantischen und pragmatischen Bereich der Müllentsorgung, die über ihre Anbringung auf den Mülleimern indiziert wird. (12, Becherbutler; 28, Kippendiener) geben konkrete Beispiele für die Gegenstände, die in den Mülleimern entsorgt werden können, und integrieren sie explizit in soziale urbane Praktiken, während (27, Beifall für Abfall; 29, Schwarzes Loch sucht Restmaterie) auf einer abstrakteren Ebene verbleibt.

\subsection{Potenzielle Referenz auf Kleinmüll in phraseologischen Ausdrücken}

Einfache und komplexe Substantive, die auf Kleinmüll referieren können, finden sich in phraseologischen, vorgeformten Ausdrücken. Man bemerkt jedoch, dass diese Verwendung stets mit Variationen einhergeht:

(30) Bin für jeden Dreck zu haben (Wien)

(31) Für die Zigarette danach (Berlin)

(32) Gib mir den Rest (Wien)

(33) Ihre Papiere bitte! (Hamburg, Wien)

Die Beispiele können noch um (9, Das Publikum gibt mir alles), (21, Darf ich Ihnen etwas abnehmen?), (22, Asche in mein Haupt) erweitert werden.

Die witzigen Mülleimeraufschriften stellen feste Redeweisen (Schlagerrefrain, Routineformeln) unverändert in einen neuen Zusammenhang (21, 29, 31), sie ersetzen die übliche 3. Person durch die erste Person (32) oder eine Richtungs- 
präposition, welche die Oberfläche eines Gegenstandes anvisiert, durch eine Richtungspräposition, die den Innenraum eines Behälters anvisiert (22, Asche in mein Haupt im Vergleich zu Asche aufs Haupt streuen).

Durch diese Veränderungen wird eine Anpassung an den konkreten Äußerungskontext erreicht (22) sowie an die EMPFÄNGER-Rolle (9, 21), die der Mülleimer sich durch den witzigen Spruch selber zuschreibt. (33) inszeniert eine fordernde Rolle: Der Mülleimer emanzipiert sich und wird aktiv. (30) ist eine Selbstaussage, um die Aufnahmebereitschaft für alle möglichen, auch ,schmutzigen“, „dreckigen“ Gegenstände klarzustellen. Insofern steht (30) in der Nähe von (19, Bin ned haglich), das ebenfalls ausdrückt, dass der Mülleimer keine Berührungsangst hat gegenüber „heiklen“ Dingen, sprich Kleinmüll.

Gemeinsam ist den phraseologischen Ausdrücken, dass sie eine doppelte Lektüre erlauben: die wörtliche und die phraseologische. Die präzise oder eher vage auf Kleinmüll verweisenden Lexeme sind dabei der Kipppunkt, der die beiden Interpretationen verbindet. Sind Asche, Zigaretten, Papier referenziell präziser als jed-Dreck, Rest, alles, etwas, so verweisen sie doch alle auf Gegenstände, die als Kleinmüll kategorisiert und in dem Mülleimer entsorgt werden können.

\subsection{Referenzpositionen rufen Prädikationen auf und umgekehrt}

Die neutralen oder witzigen Mülleimeraufschriften verbalisieren jeweils nur einige Elemente der beiden, sich mehr oder weniger überdeckenden semantischen Gesamtschemata (Bewegungs-/Übertragungsschema).

Die neutralen Mülleimeraufschriften sind wirkungsvoll, wenn sie als lokale Hinweise auf die präzisen Müllkategorien verstanden werden, die in den Behältern entsorgt werden können. Sie realisieren lediglich die denotative Position PAtiens des Bewegungsschemas. Dies setzt voraus, dass die Entsorgungshandlung als solche den semantischen und pragmatischen Hintergrund darstellt, vor dem eine punktuelle Entscheidung getroffen wird: „In welchen der Behälter werfe ich meine leere Getränkedose und das Sandwichpapier?“ Mit solchen Situationen ist jeder in verschiedenen Umgebungen konfrontiert, z.B. in Bahnhöfen, Flughäfen, Universitäten vor einer Reihe von Mülltonnen. Die Mülleimeraufschriften haben hier eine empraktische Funktion (Bühler 1978 [1934]: 155).

Die witzigen Mülleimeraufschriften haben eine andere Funktion. Zusammen mit der auffälligen Farbgebung der Behälter sind sie dazu da, die Aufmerksamkeit der Passanten zu erregen und sie dadurch daran zu erinnern, dass es Mülleimer gibt und dass man den Kleinmüll darin entsorgen kann. Es ist also nützlich, die Aufschrift so zu gestalten, dass sie zu denken gibt. Dies kann dadurch gesche- 
hen, dass Konventionen der Informativität durchbrochen werden (16, Habe durchgehend offen) oder dass Mehrdeutigkeit und Wortspiele eingesetzt werden. Aber noch ein anderer Faktor spielt eine Rolle. Die witzigen Mülleimeraufschriften sind komplex und rufen mehr als eine Position in den sich überlagernden semantischen Grundschemata auf.

Komplexe nominale Ausdrücke wie Becherbutler, Kippendiener mobilisieren explizit die Position des PATIEns (Becher, Kippe) und die des EMPFÄNGERs, dadurch also implizit die des AGENs sowie eine wie immer geartete Aktion des Übertragens. ${ }^{18}$

Eine EMPFÄNGER-zentrierte Aufschrift wie Bitte füttern fokussiert auf die Handlung und impliziert nicht nur ein AgENS sondern auch ein PATIENS, das hier konkret nur Kleinmüll sein kann. Dass die Aufschrift ein Antonym des üblichen Bitte nicht füttern! ist, das man z.B. aus Zoos kennt, ist eine Frage der Perspektivierung.

Ich versuche nun, die denotative und die sprachliche Ebene in Beziehung zu setzen.

Der Eingangssatz (A) (Ein Passant wirft einen Gegenstand in einen Müllkorb) verbalisiert eine Situation, die zum Weltwissen gehört. Die denotativen Gegebenheiten lassen sich in Gesamtschemata fassen, die sich überlagern: werfen/geben (Agens, Patiens, Ziel/Empfänger) bzw. Agens Kausativ (Patiens Bewegt Sich NACH ZIEL/EMPFÄNGER) (siehe Tab. 1).

Betrachtet man die expliziten sprachlichen Auffüllungen dieser Schemata, so bemerkt man, dass die neutralen Mülleimeraufschriften nicht die Gesamtheit der Positionen realisieren (i.). Man bemerkt ebenfalls, dass die witzigen Aufschriften zwei bis fünf Positionen der maximalen semantischen Gesamtschemata versprachlichen und perspektivieren. In iv. und v. realisieren die witzigen Müll-

18 Butler oder Diener sind Personen, die anderen gewisse Arbeiten abnehmen. Einem Diener oder Butler kann man z.B. Hut und Mantel anvertrauen, wenn man eine Wohnung oder ein Schloss betritt. Er wird sie diskret und zuverlässig an den angemessenen Ort bringen, und auch wieder rückerstatten. Letzteres ist nicht erwünscht, wenn ihm beispielsweise aufgetragen werden sollte, den Teetisch abzuräumen. Die Funktionen des zuverlässigen Entsorgens werden durch die Lexeme evoziert und verweisen diskret auf die Weiterverarbeitung des Mülls durch die städtischen Betriebe. (15, Annahmestelle, Mülleimeraufschrift in Hamburg) ebenso wie (21, Darf ich Ihnen etwas abnehmen?) gehen in die gleiche Richtung. Sie referieren auf eine Situation, in der man einer Institution oder einer Person einen Gegenstand anvertraut, damit er ihn seiner Bestimmung nach weiterleitet (z.B. einen Lottoschein) oder damit man ihn wieder zurückbekommt (z.B. einen Mantel oder eine volle Einkaufstüte). 
eimeraufschriften unterschiedliche signifikativ-semantische Perspektivierungen (werfen- vs. geben- Szenario mit den jeweiligen Komplementen und ihrer typischen Abfolge). Die witzigen Mülleimeraufschriften können ebenfalls Prädikationen über die einzelnen Elemente realisieren (in ii. und viii. z.B. den Müllbehälter), und dabei über verschiedene Mittel (z.B. Wortbildung) weitere Elemente der ZIEL-/EMPFÄNGER-Schemata mit einbeziehen. Die expliziten Elemente rufen natürlich über verschiedene Inferenzen die impliziten Elemente auf, die denotativ-semantischen Gesamtschemata dienen dabei als Interpretationsfolie. So implizieren vi. und viii. auf ganz evidente Weise die EMPFÄNGER-Rolle, ohne sie jedoch explizit zu nennen.

Die Tabelle führt die Schemapositionen nach der Perspektivierung auf, die dem Eingangssatz (A, Ein Passant wirft einen Gegenstand in einen Müllkorb) entspricht, sozusagen als Default-Perspektivierung; sie setzt somit Ziel und Empfänger gleich. Da es sich um die Modellierung der denotativen Ebene handelt, hat dies weder Vor- noch Nachteile für die signifikativ-semantische Ebene, auf der die konkreten lexikalischen und morphosyntaktischen Faktoren zum Zuge kommen.

Tab. 1: Explizite Versprachlichung der denotativ-semantischen Rollen und des Prädikats

\begin{tabular}{|c|c|c|c|c|c|}
\hline & Handlung & & Passant & Gegenstand & Müllkorb \\
\hline 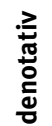 & Kausativ & $\begin{array}{l}\text { Gegenstand } \\
\text { bewegt sich }\end{array}$ & AgEns & Patiens & $\begin{array}{l}\text { ZIEL / } \\
\text { EMFÄNGER }\end{array}$ \\
\hline
\end{tabular}

\begin{tabular}{|c|c|c|c|c|c|c|c|}
\hline \multirow{8}{*}{ 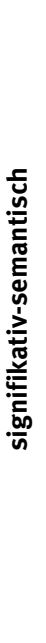 } & & Braunglas & & & & $x$ & \\
\hline & & Becherbutler & & & & $x$ & $x$ \\
\hline & iii. & $\begin{array}{l}\text { Kippen gehören } \\
\text { eingelocht! }\end{array}$ & $x$ & $x$ & & $x$ & $x$ \\
\hline & iv. & $\begin{array}{l}\text { Wirf es in den } \\
\text { Eimer, Kleines! }\end{array}$ & $x$ & $x$ & $x$ & $x$ & $x$ \\
\hline & v. & $\begin{array}{l}\text { Das Publikum } \\
\text { gibt mir alles! }\end{array}$ & $x$ & $x$ & $\times$ & $x$ & $x$ \\
\hline & vi. & $\begin{array}{l}\text { Ihre Papiere } \\
\text { bitte! }\end{array}$ & & & $x$ & $x$ & \\
\hline & vii. & $\begin{array}{l}\text { Asche in mein } \\
\text { Haupt }\end{array}$ & & $x$ & & $\times$ & $x$ \\
\hline & viii. & Annahmestelle & & $x$ & & & $x$ \\
\hline
\end{tabular}




\section{Schluss}

Mülleimer und Mülltonnen im öffentlichen Raum sind häufig beschriftet. Die Funktion der Aufschriften ist entweder empraktisch oder aufmerksamkeitserregend. Die empraktische Funktion greift in Situationen, in denen der Handelnde schon die Intention hat, Müll zu entsorgen. Die Aufschrift präzisiert dann, welcher Müll in welchen Behälter gehört. Die entsprechenden Aufschriften sind knapp, neutral, eindeutig.

Ganz anders steht es mit den witzigen Mülleimeraufschriften. Sie sind Blickfänger für den nichtintentionellen Passanten. Die Sprüche setzen Mehrdeutigkeit und Wortspiele ein, deren Auflösung genau die Zeit mobilisiert, die nötig ist, damit sich ein Aufmerksamkeitszustand einstellen kann. Das Vorhandensein eines Mülleimers soll dem Passanten bewusst werden, damit er ihn im Bedarfsfall benutzen kann. Die Funktion der witzigen Mülleimeraufschriften ist informativ und ästhetisch.

Die beiden Typen von Mülleimeraufschriften unterscheiden sich also funktional. Gemeinsam ist ihnen, dass sie auf eine Soll-Situation abheben, die im sozialen Wissen verankert ist als eine idealtypische, erwünschte Handlung: Der vorbildliche Bürger entsorgt seinen (Klein-)Müll in dem dafür vorgesehenen Behälter. Der Eingangssatz (A) beschreibt diese Situation in sehr allgemeiner, minimalistischer Weise; man kann ihn reduzieren auf Passant wirft Gegenstand in Müllkorb. Diesen Satz kann man mit semantischen Schemata erfassen, also durch die Relationen zwischen den Elementen der standardisierten Situation. Es handelt sich dabei um das BEwEGUNGS- und das EMPFÄNGER-Schema, die durch eine kausative Handlung ausgelöst werden.

Die witzigen Mülleimeraufschriften realisieren Prädikationen verschiedener Art und mit verschiedenen Perspektivierungen. Die denotative Ebene und die sprachliche Ebene sind zu trennen, spielen die witzigen Mülleimeraufschriften doch mit den vielfachen Perspektivierungsmöglichkeiten, um ihre aufmerksamkeitserregende Funktion zu erfüllen. Die Hypothese war, dass die Perspektivierungen auf mehreren Faktoren beruhen:

a) Auswahl der Positionen der semantischen Grundschemata, wobei der Rekurs auf ebendiese Grundschemata in ihrer Gesamtheit die interpretationsunterstützende Folie darstellt, auf der die Aufschrift operiert

b) Perspektivierungen wie aktiv/passiv; personal/nicht personal

c) Wortspiele.

Die Hypothese, dass sich der Unterschied zwischen neutralen und witzigen Mülleimeraufschriften unter anderem an der sprachlichen Realisierung der semantischen Grundschemata ablesen lassen könnte, hat sich im Wesentlichen bestätigt. 
Die neutralen Aufschriften sind denotativ rein PATIENS-zentriert. Dabei ist diese PATIENS-Zentrierung nur in sehr seltenen Fällen sprachlich (sprich: morphosyntaktisch) erkennbar, in den allermeisten Fällen sind die nominalen Ausdrücke für verschiedene semantische Rollen geeignet. Braunglas beispielsweise ist als Ausdruck durchaus neutral: Braunglas ist billig; aus Braunglas macht man Bierflaschen; Braunglas wird in manchen Regionen in getrennten Depotcontainern gesammelt. Es ist also das (intentionsgeleitete) Situationswissen, welches das Verständnis der neutralen nominalen Aufschrift dahin steuert, dass das Braunglas in einen bestimmten Behälter gehört.

Bei den witzigen Aufschriften hingegen überwiegen die komplexen Ausdrücke. Neben der denotativen PATIENS-Rolle werden auch andere Positionen explizit realisiert, in der Regel zwei oder mehr gleichzeitig. Es ist bemerkenswert, dass sich dies in vielen Ausdrucksformaten bestätigt. Es ist ebenso bemerkenswert, dass die witzigen Mülleimeraufschriften gelegentlich ganz auf die Gegenstandskodierung verzichten und stattdessen andere Positionen realisieren, was bei den neutralen Mülleimeraufschriften nicht möglich ist.

Eine Übersicht über die verschiedenen Realisierungen gibt Tabelle 1. Diese konzentriert sich auf die schematische Erfassung der im Eingangssatz ausgedrückten Situation durch die Mülleimeraufschriften über die explizite Realisierung der verschiedenen Positionen. Die Tabelle enthält nicht alle Fälle. Weder sind zum Beispiel Ausbuchstabierungen der Gegenstandsbewegung (24, Treffer, versenkt; 26, Man muss auch loslassen können) oder der Eigenschaften des Müllbehälters berücksichtigt, noch die eventuellen Folgen aus der richtigen Handlung (11, Beifall für Abfall) oder die Einbettung in die Sauberkeitsstrategien der Städte (13, Putzdamer Platz). Es fehlt weiterhin eine Modellierung der Inferenzen, die durch das zeit- und ortsidentische Auftreten einer bestimmten Situation und des sprachlichen Materials ausgelöst werden. Weitere Untersuchungen zu den signifikativ-semantischen Mustern wären vor allem in sprach- und kulturvergleichender Hinsicht interessant. Der Rekurs auf die denotativ-semantischen Grundschemata und ihre Ausfüllungen stellt dafür einen ersten Schritt dar.

\section{Literatur}

Ágel, Vilmos (2017): Grammatische Textanalyse. Textglieder, Satzglieder, Wortgruppenglieder. Berlin/Boston: De Gruyter.

Auer, Peter (2010): Sprachliche Landschaften. Die Strukturierung des öffentlichen Raums durch die geschriebene Sprache. In: Deppermann, Arnulf/Linke, Angelika (Hgg.): Sprache intermedial. (= Jahrbuch des Instituts für Deutsche Sprache 2009). Berlin/New York: De Gruyter. 271-298. 
Behr, Irmtraud (2005): Petite stylistique des panneaux 'régulateurs'. In: Behr, Irmtraud/ Henninger, Peter (Hgg.): A travers champs. Mélanges en l'honneur de Nicole FernandezBravo. Paris: L'Harmattan. 333-347.

Behr, Irmtraud/Lefeuvre, Florence (erscheint): Introduction. In: Faits de Langue. Numéro thématique Approche grammaticale et énonciative des genres de discours brefs.

Breitbarth, Marco/Urban, Arnd I. (2014): Littering - Ursachen, Eintragspfade, Mengen, Auswirkungen. In: Urban, Arnd I./Halm, Gerhard (Hgg.): Sauberkeit sta(d)tt Littering. (= Schriftenreihe des Fachgebietes Abfalltechnik). Kassel: University Press. 55-68.

Bühler, Karl (1978 [1943]): Sprachtheorie. Die Darstellungsfunktion der Sprache. Frankfurt a.M. u.a.: Ullstein.

Das Österreichische Wörterbuch. (www.ostarrichi.org/dictionary-search.html?search=haglich, Stand: 13.5.2017).

Digitales Wörterbuch der deutschen Sprache. (www.dwds.de, Stand: 13.5.2017).

Eggs, Friederike (2013): Wenn Mülleimer sprechen können. Eine sprach- und medienwissenschaftliche Analyse der Werbekampagne der Stadtreinigung Hamburg. In: Zeitschrift für angewandte Linguistik 59. 107-145.

Fillmore, Charles J. (1968): The case for case. In: Bach, Emmon/Harms, Robert T. (Hg.): Universals in Linguistic Theory. New York: Holt, Rinehart \& Winston. 1-88.

Köller, Wilhelm (1993): Perspektivität in Bildern und Sprachsystemen. In: Eisenberg, Peter/ Klotz, Peter (Hgg.): Sprache gebrauchen - Sprachwissen erwerben. Stuttgart u.a.: Klett Schulbuchverlag. 15-34.

Lehmann, Christian (o.J.): Situation. (www.christianlehmann.eu/ling/lg_system/sem/situation. php, Stand: 13.5.2017).

Levitte, Agnès (2013): Regards sur le mobilier urbain. Intrigues de piétons ordinaires. Paris: Le Felin.

Pörings, Ralf/Schmitz, Ulrich (2003): Sprache und Sprachwissenschaft: eine kognitiv orientierte Einführung. Tübingen: Narr. 86-95.

Welke, Klaus (2011): Valenzgrammatik des Deutschen. Berlin/New York: De Gruyter.

Wörterbuch (Ober)österreichisch - Deutsch, (Ober-) Österreichisch - Englisch. (www.fim. uni-linz.ac.at/Woerterbuch_oesterr_deut_englisch.htm, Stand: 13.5.2017).

Wunderlich, Dieter (2003): Was geschieht mit dem dritten Argument? Realisierungstypen für den Rezipienten und das Ziel. ZAS, 10. Nov. 2003. (https://user.phil.hhu.de/ wdl/ DrittesArgument.pdf, Stand: 13.5.2017).

Ziem, Alexander (2008): Frames und sprachliches Wissen. Kognitive Aspekte der semantischen Kompetenz. Berlin/New York: de Gruyter.

\section{Quellen}

Badische Zeitung (2017): Grill- und Picknicksaison: Ärger um Müll im grünen Idyll, Badische Zeitung, 26.5.2017 (www.badische-zeitung.de/freiburg/grill-und-picknicksaison-aergerum-muell-im-gruenen-idyll--135114411.html, Stand: 26.5.2017).

Geiser, Martin (2010): Warum Menschen ihren Abfall auf die Straße werfen. In: nahaufnahmen.ch, 4.9.2010. (www.nahaufnahmen.ch/2010/09/04/littering, Stand: 13.5.2017).

www.baukeinenmist.at (Stand: 26.5.2017).

Meyer, Charlotte (2016): Thermosflasche anstatt Pappbecher - Geht so eine Uni ohne Müll? (https://uni.de/redaktion/uni-ohne-muell, Stand: 13.5.2017). 
Radio Hamburg. (2017): Herzlich müllkommen! Die witzigsten Mülleimer-Sprüche Hamburgs. 27.3.2017. (www.radiohamburg.de/Nachrichten/Hamburg-aktuell/Hamburg-regional/ 2017/Maerz/Die-witzigsten-Muelleimer-Sprueche-Hamburgs, Stand: 13.5.2017).

Süddeutsche Zeitung (2017): Warum München so sauber ist, Süddeutsche Zeitung, 11. Mai 2017. (www.sueddeutsche.de/muenchen/muell-warum-muenchen-so-sauber-ist1.3495828, Stand: 13.5 .2017$)$.

SPIEGELONLINE = Töpper, Verena (2012): Kreative Müllabfuhr. Traumbilder aus der Tonne. (www.spiegel.de/karriere/fotos-mit-riesiger-lochkamera-hamburg-aus-sicht-einermuelltonne-a-840255.html, Stand: 13.5.2017). 
